\title{
Instability of Thermally Conducting Self-Gravitating Systems
}

\author{
Shaista Shaikh, Aiyub Khan \\ Department of Mathematics and Statistics, Jai Narain Vyas University, Jodhpur, India \\ E-mail: shaikh_shaista@yahoo.com \\ Received February 23, 2010; revised March 25, 2010; accepted March 25, 2010
}

\begin{abstract}
The gravitational instability of a thermally conducting self-gravitating system permeated by a uniform and oblique magnetic field has been analyzed in the framework of Tsallis' nonextensive theory for possible modifications in the Jeans' instability criterion. It is concluded that the instability criterion is indeed modified into one that depends explicitly on the nonextensive parameter. The influence of thermal conductivity on the system stability is also examined.
\end{abstract}

Keywords: Jeans’ Criterion, Nonextensivity, Thermally Conducting, Self-Gravitating

\section{Introduction}

In any subject of astrophysics and cosmology, manybody gravitating systems play an essential role. Globular clusters and elliptical galaxies, which are recognized as self-gravitating stellar systems, are typical examples. Hence, the study of stability of self-gravitating systems becomes very essential.

The condition of gravitational instability of self- gravitating systems is determined by the Jeans' criterion put forward by James Jeans [1] in 1902. In terms of wavenumber, the criterion reads: "An infinite homogenous self-gravitating atmosphere is unstable for all wavenumbers $k$ less than the Jeans' wavenumber $k_{J}=$ $\sqrt{4 \pi G \rho_{0}} / v_{S}$, where $\rho_{0}$ is the density, $G$ is the gravitational constant, $v_{S}=\sqrt{k_{B} T / m}$ is the speed of sound, $k_{B}$ is the Boltzmann's constant, $T$ is the physical temperature and $m$ is the mass of the particle."

The Jeans' problem has been extensively studied under varying assumptions. A comprehensive account of these studies has been given by Chandrasekhar [2] in his monograph on hydrodynamic and hydromagnetic stability. The Hall Effect on plasma stability has been analyzed by several researchers (Ariel [3], Bhowmik [4] and Ali \& Bhatia [5]) leading to the conclusion that Hall Currents are destabilizing in nature. Vyas \& Chhajlani [6], Sharma \& Chand [7], Khan \& Bhatia [8] have investigated the influence of permeability of porous medium on plasma instability due of the impor- tance of such studies in geology and heavy oil recovery. In view of the role played by thermally conducting fluids in various astrophysical and geophysical phenomena as well as industrial and engineering processes, the stability of such fluids has been the center of numerous analyses (Kumar [9],Chhajlani and Vaghela [10], Mehta and Bhatia [11]).

In all these investigations, the Boltzmann-Gibbs statistical mechanics have been employed to study the thermodynamics of the system. However, the physical restrictions of this formalism have been recently pointed out in different literatures based on various studies involving long-range interacting systems (Padmanabhan [12], Taruya \& Sakagami [13]). As an alternative, the nonextensive theory proposed by Tsallis [14] is gaining considerable attention.

The new framework for thermodynamics based on Tsallis' nonextensive theory has been applied extensively to deal with a variety of interesting problems to which the standard B-G statistical mechanics cannot be applied. Examples include the study of waves and instability phenomena, such as the plasma oscillations $[15,16]$, the relativistic Langmuir waves [17], the linear or nonlinear Landau damping in plasmas [18] and dark matter and gas density profiles observed in galaxies and clusters [19]. The study of self-gravitating stellar systems has been one of the most interesting applications of Tsallis' nonextensive thermodynamics [20-24].

In this paper, we analyze the stability of a thermally conducting self-gravitating system embedded by a uniform and oblique magnetic field for possible modification 
in the Jeans' instability criterion due to the presence of nonextensive effects. The influence of thermal conductivity on the growth rate of the system is also examined.

\section{Nonextensive Theory}

The physical restrictions of the Boltzmann-Gibbs statistical mechanics have stressed the need for a possible generalization of this formalism. Such a generalization was proposed by Tsallis in 1988 (known as “Tsallis' Statistics”) by constructing a new form of entropy written as

$$
S_{q}=k_{B}\left(\sum_{i} p_{i}^{q}-1\right) /(1-q)
$$

where $p_{i}$ is the probability of the $i$ th microstate and $q$ is a parameter quantifying the degree of nonextensivity of the system. In the limit $q \rightarrow 1$, the celebrated B-G extensive formula, namely

$$
S=-k_{B} \sum_{i} p_{i} \ln p_{i}
$$

is recovered.

Various literatures involving the thermo-statistical analysis of many astrophysical systems and processes (Plastino \& Plastino [25], Abe [26]) make it clear that Tsallis' statistics may be the appropriate theory for description of astrophysical systems with long-range interaction of gravitation.

The nonextensivity in the Jeans' problem is introduced through the equation of state of an ideal gas. In the framework of nonextensive theory, the $q$-nonextensive velocity distribution function for free particles is given by

$$
f(v)=B_{q}\left[1-(1-q) \frac{m v^{2}}{2 k_{B} T}\right]^{\frac{1}{1-q}}
$$

where $B_{q}$ is a normalization constant and the other variables have their usual meanings.

If $N$ denotes the particle number density, pressure is defined by $P=\frac{1}{3} N m<v^{2}>$ with $\left\langle v^{2}>\right.$ the mean square velocity of the particle defined in Tsallis' statistics by

$$
<v^{2}>=\frac{\int v^{2}[f(v)]^{q} d^{3} v}{\int[f(v)]^{q} d^{3} v}
$$

In 2003, Silva \& Alcaniz [27] calculated the $q$ expectation value for the square velocity of the particle as

$$
<v^{2}>_{q}=\frac{6}{5-3 q} \frac{k_{B} T}{m},(0<q<5 / 3)
$$

Clearly, the standard mean square velocity $<v^{2}>=3 k_{B} T / m$ is perfectly recovered when $q \rightarrow 1$. Thus, the equation of state of an ideal gas in the nonextensive kinetic theory is obtained as

$$
P_{q}=\frac{1}{3} N m<v^{2}>_{q}=\frac{2}{5-3 q} \frac{k_{B} \rho T}{m}
$$

where we have written $N=\rho / m$. Note that the standard equation of state is correctly recovered in the limit $q \rightarrow 1$. The above equation can also be written in the form $P_{q}=N k_{B} T_{q}$, with the physical temperature $T_{q}$, a variable that depends on the nonextensive parameter $q$ as $T_{q}=\frac{2 T}{5-3 q}$. Consequently, the speed of sound can be written as

$$
S_{q}=\sqrt{\frac{k_{B} T_{q}}{m}}=S \sqrt{\frac{2}{5-3 q}}
$$

significantly different from the one in B-G statistics ( $q$ $\left.=1, T_{q}=T\right)$. We shall use this modified form while writing the perturbation equations of the self-gravitating system considered in this paper.

\section{Perturbation Equations}

Following standard lines, we write the linearized perturbation equations characterizing the flow of a thermally conducting self-gravitating fluid embedded by a uniform and oblique magnetic field denoted by $\vec{H}=\left(H_{x}, 0, H_{z}\right)$.

$$
\begin{gathered}
\frac{\partial \rho_{1}}{\partial t}+\rho \nabla \cdot \vec{u}=0 \\
\frac{\partial \vec{u}}{\partial t}=-\frac{1}{\rho} \nabla p_{1}-\nabla \phi_{1}+\left(\nabla \times \overrightarrow{h_{1}}\right) \times \vec{H} \\
\frac{\partial \overrightarrow{h_{1}}}{\partial t}=\nabla \times(\vec{u} \times \vec{H}) \\
\nabla^{2} \phi_{1}=-G \rho_{1} \\
\frac{\partial}{\partial t}\left(p_{1}-\gamma S_{q}^{2} \rho_{1}\right)=\gamma \chi \nabla^{2}\left(p_{1}-S_{q}^{2} \rho_{1}\right)
\end{gathered}
$$

where $\vec{u}(u, v, w), \vec{h}_{1}\left(h_{X}, h_{y}, h_{z}\right), \rho_{1}, p_{1}$ and $\phi_{1}$ are respectively the perturbations in velocity $\vec{u}$, magnetic field $\vec{H}$, density $\rho$, pressure $\rho$ and gravitational potential $\phi$, $G$ is the gravitational constant, $\gamma$ denotes an adiabatic exponent and $x$ is the coefficient of thermal conductivity.

We seek the solutions of the Equations (8)-(12) whose dependence on the space coordinates $(x, y, z)$ and time $t$ is of the form

$$
\exp (i k \sin \theta . x+i k \cos \theta . z+i \omega . t)
$$

where $\vec{k}=(k \sin \theta, 0, k \cos \theta)$ is the wavenumber of perturbation making angle $\theta$ with the x-axis and $\omega$ is the frequency of perturbation. Eliminating $\rho_{1}, \phi_{1} \& p_{1}$ from the above equations, we get six equations govern- 
ing the perturbation of velocity and magnetic field which can be written in the matrix form

$$
[A][B]=0
$$

where $[A]$ is a sixth order square matrix and $[B]$ is a single column matrix in which the elements are $\left(u, v, w, h_{x}, h_{y}, h_{z}\right)^{T}$. The elements of $[A]$ are

$$
\begin{aligned}
& A_{11}=i \omega-i D \sin ^{2} \theta, \\
& A_{12}=0, A_{13}=(-i D) \sin \theta \cos \theta, \\
& A_{14}=-\frac{H}{\rho} i k \cos \theta, A_{15}=0, A_{16}=\frac{H}{\rho} i k \sin \theta, \\
& A_{21}=0, A_{22}=i \omega, A_{23}=0, \\
& A_{24}=0, A_{25}=-\frac{i k}{\rho}\left(H_{x} \sin \theta+H_{z} \cos \theta\right), A_{26}=0, \\
& A_{31}=(-i D) \sin \theta \cos \theta, A_{32}=0, A_{33}=i \omega-i D \cos ^{2} \theta, \\
& A_{34}=\frac{H_{x}}{\rho} i k \cos \theta, A_{55}=0, A_{36}=-\frac{H_{x}}{\rho} i k \sin \theta, \\
& A_{41}=-H_{z} i k \cos \theta, A_{42}=0, A_{43}=H_{x} i k \cos \theta, \\
& A_{44}=i \omega, A_{45}=0, A_{46}=0, \\
& A_{51}=0, A_{52}=-i k\left(H_{x} \sin \theta+H_{z} \cos \theta\right), A_{53}=0, \\
& A_{54}=0, A_{55}=i \omega, A_{56}=0, \\
& A_{61}=H_{z} i k \sin \theta, A_{62}=0, A_{63}=-H_{x} i k \sin \theta, \\
& \quad A_{64}=0, A_{65}=0, A_{66}=i \omega
\end{aligned}
$$

where we have written

$$
D=\frac{S_{q}^{2} k^{2} \gamma\left(i \omega+\chi k^{2}\right)-G \rho\left(i \omega+\gamma \chi k^{2}\right)}{\omega\left(i \omega+\gamma \chi k^{2}\right)}
$$

\section{Dispersion Relation}

The vanishing of $|A|$ gives the dispersion relation as the product of three factors:

$(i \omega) \cdot\left\{(i \omega)^{2}+k^{2} V^{2}\right\} \cdot\left\{(i \omega)^{2}(i \omega-i D)+(i \omega) \frac{\left(H_{x}^{2}+H_{z}^{2}\right)}{\rho} k^{2}-i D k^{2} V^{2}\right\}$

By writing $\omega=i n$ and using the value of $D$ in the third factor of Equation (17), we obtain the resulting dispersion relation, which is an equation of degree five in $n$ of the form

$$
n^{5}-c_{4} n^{4}+c_{3} n^{3}-c_{2} n^{2}+c_{1} n-c_{0}=0
$$

with the coefficients $c_{4}$ to $c_{0}$ given by

$$
\begin{aligned}
& c_{4}=\gamma \chi k^{2} \\
& c_{3}=S_{q}^{2} k^{2} \gamma-G \rho-\frac{\left(H_{x}^{2}+H_{z}^{2}\right)}{\rho} k^{2} \\
& c_{2}=\gamma \chi k^{2}\left(S_{q}^{2} k^{2}-G \rho-\frac{\left(H_{x}^{2}+H_{z}^{2}\right)}{\rho} k^{2}\right) \\
& c_{1}=k^{2} V^{2}\left(S_{q}^{2} k^{2} \gamma-G \rho\right) \\
& c_{0}=\gamma \chi k^{4} V^{2}\left(S_{q}^{2} k^{2}-G \rho\right)
\end{aligned}
$$

where we have taken

$$
V^{2}=\frac{\left(H_{x} \sin \theta+H_{z} \cos \theta\right)^{2}}{\rho}
$$

\section{Analysis of Dispersion Relation}

In the study of Jeans' instability, the boundary between stable and unstable solutions is achieved by setting $n=0$ in the dispersion relation (Equation (17)). The result is a family of $q$-parameterized critical wavenumbers $k_{q}$ given by

$$
k_{q}=\frac{\sqrt{G \rho}}{S_{q}}=k_{J} \sqrt{\frac{5-3 q}{2}}
$$

Note that the standard values as obtained from fluid theory are recovered only if $q=1$. We have, thus, obtained a modified form of Jeans' Criterion which shall now be analyzed for different values of $q$. As we know, the value of nonextensive parameter $q$ lies between 0 and 5/3. Hence, we will analyze the Jeans' criterion for different values of $q$ in this range. Let us calculate the critical wave numbers for $q=1, q=0.3$, i.e. $0<q<1$ and $q=1.6$, i.e. $1<q<5 / 3$. For these calculations, we take numerical values for conditions prevailing in magnetic collapsing clouds:

$$
\begin{gathered}
\rho=1.7 \times 10^{-21} \mathrm{kgm}^{-3}, \\
G=6.658 \times 10^{-11}(\mathrm{~kg})^{-1} \mathrm{~m}^{3} \mathrm{~s}^{-2}, \\
S^{2}=2.5 \times 10^{8} \mathrm{~m}^{2} \mathrm{~s}^{-2}, \\
V^{2}=5 \times 10^{8} \mathrm{~m}^{2} \mathrm{~s}^{-2} .
\end{gathered}
$$

The following critical wave numbers are obtained through numerical calculations

$$
\begin{aligned}
& k_{q=1.0}=2.12 \times 10^{-20} \mathrm{~m}^{-1} \\
& k_{q=0.3}=3.04 \times 10^{-20} \mathrm{~m}^{-1} \\
& k_{q=1.6}=0.67 \times 10^{-20} \mathrm{~m}^{-1}
\end{aligned}
$$


Let us discuss the Jeans' Criterion in light of the above.

a) When $q=1$, the Jeans' Criterion as obtained through fluid theory is recovered perfectly. The system is unstable for wavenumbers $k<k_{q=1.0}$ and stable for wavenumbers $k>k_{q=1.0}$.

b) When $q=0.3$ i.e. $0<q<1$, the system is unstable for $k<k_{q=0.3}$ and stable for wave numbers $k>k_{q=0.3}$. Hence, the Jeans' Criterion is modified as $k<k_{q=0.3}$ and $k>k_{q=1.0}$ i.e. the system may now be unstable even for the wave numbers greater than $k_{q=1.0}$ provided that they are less than $k_{q=0.3}$.

c) When $q=1.6$ i.e. $1<q<5 / 3$, the system is unstable for $k<k_{q=1.6}$ and stable for wave numbers $k>k_{q=1.6}$. Hence, the Jeans' Criterion is modified as $k>k_{q=1.6}<k_{q=1.0}$ i.e. the system which was believed to be unstable for wave numbers less than $k_{q=1.0}$ according to fluid theory, may now be stable for wave numbers less than $k_{q=1.0}$ but greater than $k_{q=1.6}$.

We have demonstrated the effect of nonextensive parameter $q$ on the system stability by plotting wavenumber against growth rate for the values of $q$ mentioned above. The result is as shown in Figure 1. The same conclusions, as outlined in a)-c), are drawn by studying the plot.

In order to gauge the influence of thermal conductivity on the growth rate of the system, we have plotted wavenumber against growth rate for varying values of thermal conductivity in Figure 2 for a fixed value of nonextensive parameter $q=1$. We notice that as the value of $\chi$ (taken as $\mathrm{X}$ in the figure) increases, the value of growth rate initially increases in the unstable region. However, as the system moves from unstable to stable region, the growth rate decreases with increase in thermal conductivity for a fixed wave number. Hence, we conclude that thermal conductivity has a mixed, but predominantly stabilizing, influence on the system stability.

\section{Results}

The Jeans' gravitational instability of a thermally conducting self-gravitating fluid permeated by a uniform and oblique magnetic field has been analyzed in the framework of nonextensive theory. It is concluded that thermal conductivity has a predominantly stabilizing influence on the growth rate of the system. The presence of nonextensive effects modifies the standard Jeans' Criterion into one that depends explicitly on the nonextensive parameter $q$. However, in spite of this modification, the basic instability criterion is maintained: perturbations with $k>k_{q}$ do not grow while instability takes place for $k<k_{q}$.

\section{Concluding Remarks}

We have studied the stability of a large-scale self- gravitating system in the framework of Tsallis' Nonextensive Statistical Mechanics (NSM). Our approach differs from the kinetic theoretical approach based on the Vlasov equation, where the evolution of the system is described by perturbing the equilibrium Max-wellian velocity distribution function. We have, instead, considered the non-Maxwellian (power-law) equilibrium distribution function (Equation (3)) which is a nonextensive generalization of the standard distribution function. Considerable

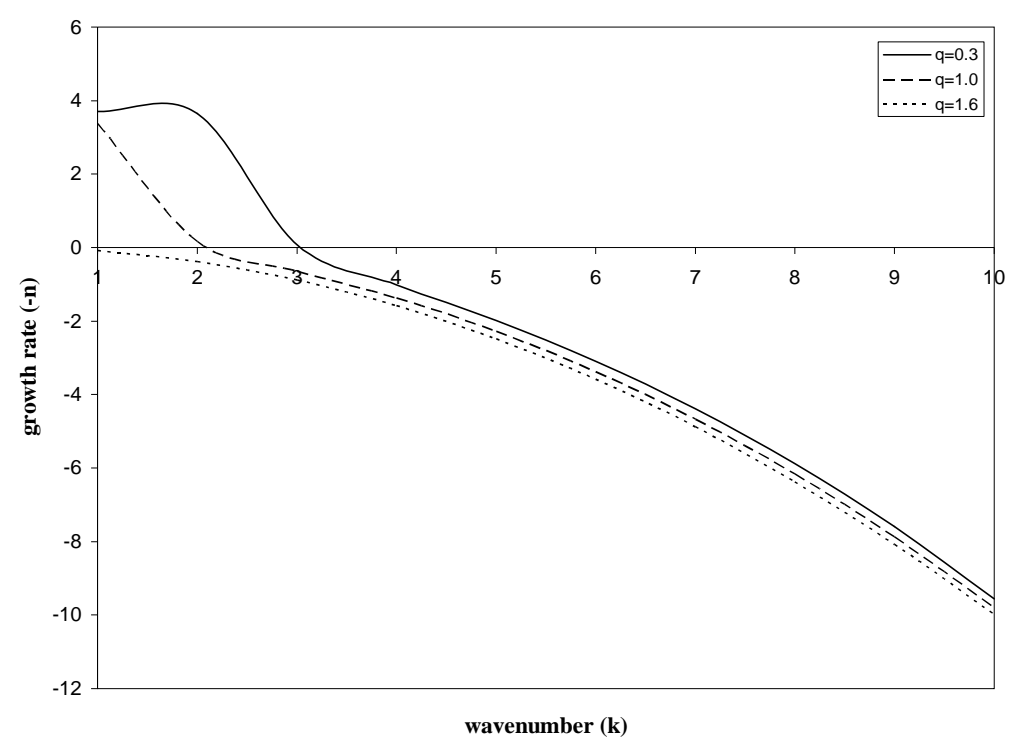

Figure 1. Graph of wavenumber vs. growth rate of a thermally conducting fluid for varying values of nonextensive parameter. 


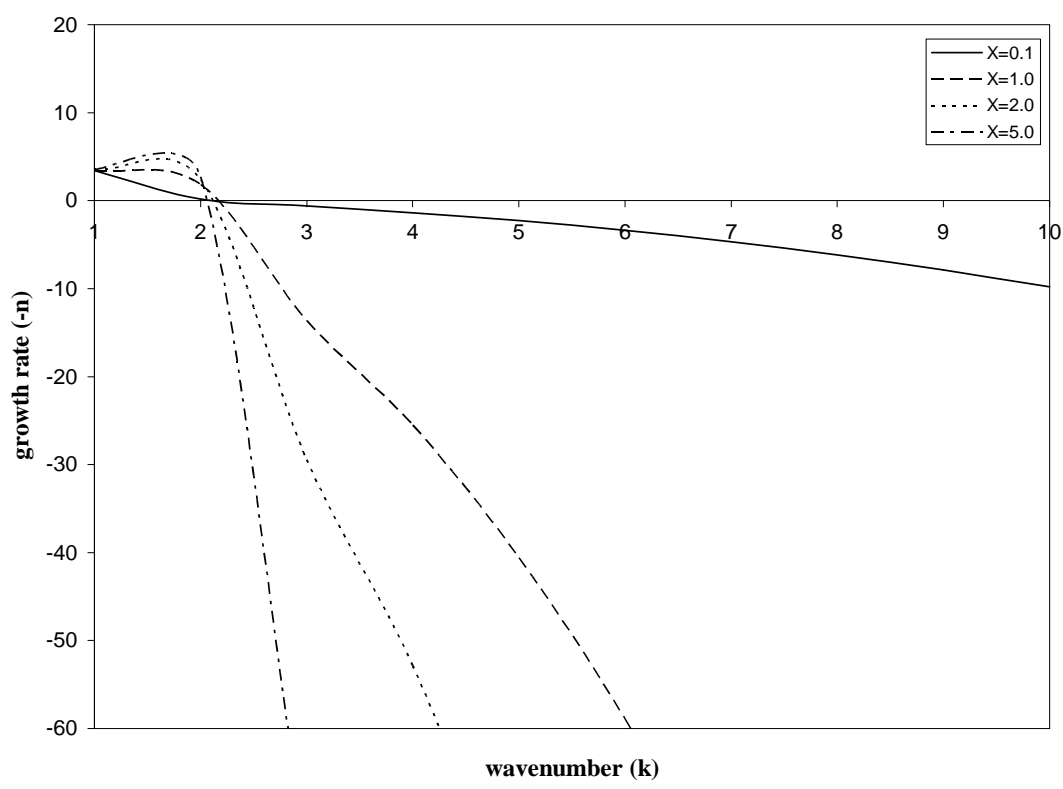

Figure 2. Graph of wavenumber vs. growth rate of a thermally conducting fluid for varying values of coefficient of thermal conductivity.

amount of experimental evidence supports the employment of such a distribution (e.g. Liu et al. [28]), clearly indicating that the standarsd Maxwellian velocity distribution might provide only a very crude description of the velocity distribution for a self-gravitating gas, or generally for any system endowed with long range interactions. Infact, a well determined criterion for gravitational instability is not a privilege of the exponential velocity distribution function, but is shared by an entire family of power-law functions (named q-exponentials) which includes the standard Jeans' result for the Maxwellian distribution as a limiting case $(q=1)$. This being said, it must also be stressed that all nonextensive systems need not require the Tsallis' statistics to understand their behaviors (Cohen [29]). In the light of present understanding, it is still unclear which class of nonextensive systems requires Tsallis' statistics for its statistical description, mainly due to the fact that the physical meaning of the nonextensive parameter $q$ is yet to be settled. Although some progress in this regard is being made $(\mathrm{Du}$ $[22,23])$, the Nonextensive Statistical Mechanics remains open to further verification and deeper understanding.

Further Reading: Interested readers may refer to similar works by the authors $[30,31,32]$ on the instability of thermally conducting self-gravitating systems in the framework of nonextensive statistics.

Acknowledgements: This work was carried out as part of a major research project (F. No. 36-103/2008 (SR)) awarded by University Grants Commission (UGC), India to the one of the authors, Aiyub Khan. The financial assistance from UGC is gratefully acknowledged. The authors are also grateful to the reviewer for useful suggestions, which have helped in improving the overall presentation and quality of the paper.

\section{References}

[1] J. H. Jeans, “The Stability of Spherical Nebulae,” Philosophical Transactions of the Royal Society of London, A 199, 1902, pp. 1-53.

[2] S. Chandrasekhar, "Hydrodynamics \& Hydromagnetic Stability," Clarendron Press, Oxford, 1961.

[3] P. D. Ariel, "The Character of Equilibrium of an Inviscid Infinitely Conducting Fluid of Variable Density in the Presence of a Horizontal Magnetic Field with Hall-Urrent," Journal of Plasma Physics, Vol. 4, 1970, pp. 523-530.

[4] G. Bhowmik, "Rayleigh Taylor Instability of a Viscous Hall Plasma with Magnetic Field,” Journal of Plasma Physics, Vol. 7, 1972, pp. 117-132.

[5] A. Ali and P. K. Bhatia, "Magnetic Resistivity and Hall Currents Effects on the Stability of a Self-Gravitating Plasma of Varying Density in Variable Magnetic Field," Astrophysics and Space Science, Vol. 201, 1993, pp. 15-27.

[6] M. K. Vyas and R. K. Chhajlani, "Gravitational Instability of a thermally-conducting Plasma Flowing through a Porous Medium in the Presence of Suspended Particles," Astrophysics and Space Science, Vol. 149, 1988, pp. 323-342.

[7] R. C. Sharma and T. Chand, "Gravitational Instability for Some Astrophysical Systems," Astrophysics and Space Science, Vol. 183, 1991, pp. 215-224.

[8] A. Khan and P. K. Bhatia, "Stability of Two Superposed Viscoelastic Fluid in a Horizontal Magnetic Field," Indian Journal of Pure and Applied Mathematics, Vol. 32, 2001, pp. 98-108.

[9] S. S. Kumar, “On Gravitational Instability, III,” Publications of the Astronomical Society of Japan, Vol. 13, 1961, 
pp. 121-124.

[10] R. H. Chhajlani and D. S. Vaghela, "Magnetogravitational Stability of Self-Gravitating Plasma with Thermal Conduction and Finite Larmor Radius through Porous Medium,” Astrophysics and Space Science, Vol. 134, 1987, pp. 301315.

[11] V. Mehta and P. K. Bhatia, "Gravitational Instability of a Rotating Viscous Thermally Conducting plasma,” Contributions to Plasma Physics, Vol. 29, 1989, pp. 617-626.

[12] T. Padmanabhan, "Statistical Mechanics of Gravitating Systems,” Physics Reports, Vol. 188, 1990, pp. 285-362.

[13] A. Taruya and M. Sakagami, "Thermodynamic Properties of Stellar Polytrope,” Physica A, Vol. 318, 2003, pp. 387-413.

[14] C. Tsallis, "Possible Generalization of Boltzmann-Gibbs Statistics,” Journal of Statistical Physics, Vol. 52, 1988, pp. 479-487.

[15] R. Silva, J. A. Alcaniz and J. A. S. Lima, "Constraining Nonextensive Statistics with Plasma Oscillation Data," Physica A, Vol. 356, 2005, pp. 509-516.

[16] S. Shaikh, A. Khan and P. K. Bhatia, “Jeans’ Gravitational Instability of a Thermally Conducting, Unbounded, Partially Ionized Plasma," Zeitschrift für Naturforschung, Vol. 61, 2006, pp. 275-280.

[17] V. Munoz, "A Nonextensive Statistics Approach for Langmuir Waves in Relativistic Plasmas," Nonlinear Processes in Geophysics, Vol. 13, 2006, pp. 237-241.

[18] F. Valentini, "Nonlinear Landau Damping in Nonexten-sive Statistics," Physics of Plasmas, Vol. 12, 2005, pp. 1-7.

[19] M. P. Leubner, "Nonextensive Theory of Dark Matter and Gas Density Profiles,” The Astrophysical Journal, Vol. 632, 2005, pp. L1-L4.

[20] J. A. S. Lima, R. Silva and J. Santos, “Jeans’ Gravitational Instability and Nonextensive Kinetic Theory," Astronomy and Astrophysics, Vol. 396, 2002, pp. 309-313.

[21] J. L. Du, “Jeans' Criterion and Nonextensive Velocity Distribution Function in Kinetic Theory," Physics Letters A, Vol. 320, 2004, pp. 347-351.
[22] J. L. Du, "Nonextensivity in Nonequilibrium Plasma Systems with Coulombian Long-Range Interactions," Physics Letters A, Vol. 329, 2004, pp. 262-267.

[23] J. L. Du, "What Does the Nonextensive Parameter Stand for in Self-Gravitating Systems?” Astrophysics and Space Science, Vol. 305, 2006, pp. 247-251.

[24] J. L. Du, "Nonextensivity and the Power-Law Distributions for the Systems with Self-Gravitating Long-Range Interactions,” Astrophysics and Space Science, Vol. 312, 2007, pp. 47-55.

[25] A. Plastino and A. R. Plastino, "Stellar Polytropes and Tsallis’ Entropy,” Physics Letters A, Vol. 174, 1993, pp. 384-386.

[26] S. Abe, "Thermodynamic Limit of a Classical gas in Nonextensive Statistical Mechanics: Negative Specific Heat and Polytropism,” Physics Letters A, Vol. 263, 1999, pp. 424-429.

[27] R. Silva and J. S. Alcaniz, "Negative Heat Capacity and Non-Extensive Kinetic Theory,” Physics Letters A, Vol. 313, 2003, pp. 393-396.

[28] J. M. Liu, J. S. D. Groot, J. P. Matte, T. W. Johnston and R. P. Drake, "Measurements of Inverse Bremsstrahlung Absorption and Non-Maxwellian Electron Velocity Distributions,” Physical Review Letters, Vol. 72, 1994, pp. 2717-2720.

[29] E. G. D. Cohen, "Statistics and Dynamics," Physica A, Vol. 305, 2002, pp. 19-26.

[30] S. Shaikh, A. Khan and P. K. Bhatia, "Stability of Thermally Conducting Plasma in a Variable Magnetic Field," Astrophysics and Space Science, Vol. 312, 2007, pp. 35-40.

[31] S. Shaikh, A. Khan and P. K. Bhatia, "Thermally Conducting Partially Ionized Plasma in a Variable Magnetic Field,” Contributions to Plasma Physics, Vol. 47, No. 3, 2007, pp. 147-156.

[32] S. Shaikh, A. Khan and P. K. Bhatia, “Jeans' Gravitational Instability of a Thermally Conducting Plasma,” Physics Letters A, Vol. 372, 2008, pp. 1451-1457. 